\title{
CARDIOPULMONARY EXERCISE TEST IN EVALUATION OF FUNCTIONAL CAPACITY IN PATIENTS WITH CHRONIC OBSTRUCTIVE PULMONARY DISEASE
}

\author{
KHATUN H ${ }^{1}$, RAHMAN MM ${ }^{2}$, CHOWDHURY HA ${ }^{3}$, ISLAM MA ${ }^{4}$, TOUHIDUZZMAN ${ }^{5}$, AHMAD \\ $\mathrm{MM}^{6}$, HASSAN MR ${ }^{7}$, ASHRAFUZZAMAN ${ }^{8}$, AHMED MAU ${ }^{9}$, HOSSAIN MZ $^{10}$
}

\begin{abstract}
Background: Assessment of severity in patients with Chronic Obstructive Pulmonary Disease $(C O P D)$ is usually done at rest by spirometry test. But cardiopulmonary exercise test can be a measure to assess the functional capacity more accurately in patients with COPD.

Method: Fifty eight patients with stable COPD were included in this study period of one and half years from January 15 to June 2016 and categorise into four groups based on spirometric data following the GOLD guidelines 2018; GOLD 1 mild COPD, GOLD 2 moderate COPD, GOLD 3 severe COPD and GOLD 4 very severe COPD. Symptom limited CPET on treadmill using Bruce protocol using cardiopulmonary exercise machine was done in all fifty eight patient.

Results: twenty one patients (36.20\%) had moderate COPD; twenty six(44.82\%) had severe COPD; eleven(18.96\%) had very severe COPD and none found to be categorised as mild COPD. All fifty eight patients attained anaerobic threshold. Majority(34, 58.62\%) of patients stopped exercise due to shortness of breath; decreased oxygen saturation was the cause of stopping exercise in eight(13.7\%) patients; other causes of exercise limitation were increased heart rate, vertigo, leg fatigue, increased blood pressure, ventricular ectopic and chest tightness. Four patients were found to limit exercise due to combined shortness of breath and leg fatigue.

A significant correlation between forced expiratory volume in first second (FEV1, percent predicted) and maximum oxygen uptake $(\mathrm{ml} / \mathrm{kg} / \mathrm{min})$ was observe.

Twenty(46.55\%) patients with low(<40\%) AT were identified who could be benefited from pulmonary rehabilitation program.

Conclusion: CPET can be useful to identifying the COPD patients with functional capacity impairment for pulmonary rehabilitation by assessing exercising capacity and the cause of exercise limitation.
\end{abstract}

Key words : cardiopulmonary exercise test, functional capacity, chronic obstructive pulmonary disease.

J Dhaka Med Coll. 2019; 28(1) : 23-27

\section{Introduction}

Functional Capacity impairment is one of the major indicator of the disease severity in chronic obstructive pulmonary disease (COPD) patient. It predicts exacerbation ${ }^{1}$, hospital admission ${ }^{2}$ and mortality $1,2,3$. Functional Capacity measurement is also very useful for objective evaluation of outcome of treatment intervention. Exercise training is an integral component of comprehensive pulmonary rehabilitation in

1. Dr. Hena Khatun, Assistant Professor, Department of Respiratory Medicine, Dhaka Medical College, Dhaka.

2. Dr. Mohammad Mostafizur Rahman, Officer on Special Duty, Department of Physiology, Dhaka Medical College, Dhaka.

3. Dr. Hasina Akhter Chowdhury, Research Coordinator, Centre for Injury Prevention and Research, Bangladesh

4. Dr. Mohammad Aminul Islam, Associate Professor, Department of Respiratory Medicine, Dhaka Medical College, Dhaka.

5. Dr. Mohammad Touhiduzzaman, Assistant Professor, Department of Respiratory Medicine, Dhaka Medical College, Dhaka.

6. Dr. Mohammad Mohiuddin Ahmad, Professor, Department of Respiratory Medicine, Dhaka Medical College, Dhaka.

7. Dr. Md. Rashidul Hassan, Professor, Respiratory Medicine, Anwer Khan Modern Medical College, Dhaka.

8. Dr. Md. Ashrafuzzaman, Registrar, Unit -3, NIDCH, Mohakhali, Dhaka

9. Dr. Md. Ashraf Uddin Ahmed, Resident Physician, BIRDEM General Hospital, Dhaka

10. Dr. Md. Zakir Hossain, Junior Consultant, Department of Medicine, UHC, Keranigang, Dhaka

Correspondence : Hena Khatun, Assistant Professor, Department of Respiratory Medicine, Dhaka Medical College, Dhaka. 
patients with $\mathrm{COPD}^{4}$. Functional Capacity measurement is necessary before exercise training for exercise prescription. Then after training the measurement is done again to objectively document the improvement and to refine training levels.

At present, we usually assess disease severity in COPD patient mainly with spirometry at resting condition. But as the functional level of a patient is the integrated influence of multiple factors, e.g. pulmonary mechanics, pulmonary circulation, cardiac function, peripheral muscle and psychological factors; which can be better assessed by dynamic exercise tests. These exercise tests include cardiopulmonary pulmonary exercise test (CPET), Shuttle Walk Test, 6 Minute Walk Test, Sit to Stand Test, Stair Climbing Test etc 6,7,8,9.

CPET is unparalleled compared with other exercise test modalities. This relatively noninvasive, dynamic physiologic overview permits the objective determination of both submaximal and peak exercise responses, providing the physician with relevant information for clinical decision making. CPET provides a global assessment of the integrative exercise responses involving the pulmonary, cardiovascular, blood, brain and skeletal muscle system, which are not adequately reflected through the measurement of individual organ system function. In patient with COPD, maximal oxygen uptake $\left(\mathrm{Vo}_{2} \max \right)$ has been considered to reflect the severity of the disease in contrast to resting pulmonary function test which cannot predict exercise performance and exercise induced hypoxaemia ${ }^{10}$. This accurate assessment of severity and functional capacity with CPET in patients of COPD may be useful for purpose of exercise prescription and determining response to therapy.

\section{Materials and methodology:}

This Observational analytical study was carried out at National Institute of Diseases of the Chest and Hospital (NIDCH) Dhaka, Bangladesh, from January 2015 to June 2016, with a primary objective to evaluate the functional capacity of
COPD patients, to determine the cause of exercise limitation and to correlate $\mathrm{FEV}_{1}$ and $\mathrm{Vo}_{2}$ max by CPET.

Prior to the commencement of this study approval of the research protocol was taken from the institutional research ethical committee of NIDCH. It was performed in accordance with the ethical standards of the Helsinki Declaration (1964, amended in 1975 and 1983) of the world medical association. All participants read and signed the terms consent before being included in the study.

Inclusion Criteria: The patients were clinically stable cases of COPD attending Pulmonary Medicine outpatient department between the age group of 50-75 years of either gender, with duration of symptoms for at least 5 years, irrespective of smoking status and spirometry confirmed COPD Gold category I-IV, were enrolled. The exclusion criteria adopted were: acute or chronic cardiac disease, patient on long term oxygen therapy, patient with arterial oxygen saturation less than $88 \%$ at rest and with any musculoskeletal and neurological disorder that will hinder exercise and other pulmonary disease or comorbid condition that may limit physical exercise. Patient's detailed history, baseline demographic data and disease severity evaluation were recorded in a predesigned proforma.

Resting pulmonary function test is done by spirometry with reversibility test as recommended by the American Thoracic Society ${ }^{11}$ and patients were categorised into four groups based on spirometric data following GOLD guidelines 2018; GOLD 1: mild COPD, GOLD 2: moderate COPD, GOLD 3: severe COPD and GOLD 4: very severe COPD.

Symptom limited CPET was performed on treadmill using Bruce protocol through cardiopulmonary exercise machine (Ultima TM system, model: Ultima GX) in all fifty-eight patients.

Resting pulmonary function test and CPET were performed on the same day as per ATS guidelines. 
Oxygen uptake $\left(\mathrm{Vo}_{2}\right)$ is volume of $\mathrm{O}_{2}$ extract from inspired air in a given period of time, expressed in millilitres per $\mathrm{kg}$ per minute. Maximal oxygen uptake $\left(\mathrm{Vo}_{2} \max \right)$ is the highest attainable $\mathrm{O}_{2}$ uptake for a given subject. It is determined during dynamic exercise from a 'plateauing' of $\mathrm{Vo}_{2}$ despite work rate continuing to increase.

Anaerobic threshold (AT) was defined as: (i) the point at which the ventilatory equivalent for $\mathrm{O}_{2}\left(\mathrm{VE} / \mathrm{Vo}_{2}\right)$ was minimal followed by a progressive increase; (ii) the point after which the respiratory gas exchange ratio consistently exceeded 1; and (iii) the $\mathrm{Vo}_{2}$ after which expired carbon dioxide increased non-linearly relative to oxygen consumption. The anaerobic threshold percent $(\% \mathrm{AT})$ was defined as $\left(\mathrm{Vo}_{2}\right.$ at $\mathrm{AT} /$ predicted maximal $\mathrm{Vo}_{2}$ ) $\times 100$.

\section{Statistical analysis}

Data were processed and analysed by using SPSS (statistical Package for Social Science), version 16 software. Pearson's correlation coefficient test was used at a level of significance p-value of less than 0.05 .

\section{Results}

A total of 58 patients were enrolled in this study, of them $55(94.8 \%)$ were male and $3(5.2 \%)$ were female.

\section{Table-I}

Anthropometric and pulmonary function data $(n=58)$

\begin{tabular}{lcc}
\hline Parameter & Mean \pm SD & Range \\
\hline Age $($ years $)$ & $60.24 \pm 9.32$ & $41-75$ \\
$\mathrm{BMI}\left(\mathrm{kg} / \mathrm{m}^{2}\right)$ & $20.90 \pm 4.43$ & $12.61-32.27$ \\
$\mathrm{FVC}(\%$ predicted $)$ & $59.94 \pm 14.03$ & $19-87$ \\
$\mathrm{FEV}_{1}(\%$ predicted $)$ & $44.87 \pm 14.95$ & $19-79$ \\
$\mathrm{FEV}_{1} / \mathrm{FVC}$ & $55.76 \pm 10.53$ & $33-69$ \\
$\mathrm{Vo}_{2} \mathrm{max}(\mathrm{ml} / \mathrm{kg} / \mathrm{min})$ & $17.96 \pm 6.54$ & $9-38$ \\
$\mathrm{AT}(\%)$ & $43.86 \pm 17.62$ & $17-105$ \\
\hline
\end{tabular}

Table-II

Distribution of COPD patients according to GOLD guideline $(n=58)$

\begin{tabular}{lcccc}
\hline GOLD & GOLD 1 & GOLD 2 & GOLD 3 & GOLD 4 \\
\hline Category & Mild COPD & Moderate COPD & Severe COPD & Very Severe COPD \\
& FEV1 $\geq 80 \%$ & FEV1 50-79\% & FEV1 30-49\% & FEV1 $<30 \%$ \\
& predicted & predicted & predicted & predicted \\
No. of Patients & 0 & $21(36.20 \%)$ & $26(44.82 \%)$ & $11(18.96 \%)$ \\
\hline
\end{tabular}

Fifty-eight patients completed spirometry with reversibility test and CPET. Twenty-one patients $(36.20 \%)$ had moderate COPD; twenty-six (44.82\%) had severe COPD; eleven (18.96\%) had very severe COPD and none were found to be categorised as mild COPD.

Table-III

$\mathrm{VO}_{2}$ max and AT in different GOLD category of COPD patients. ( $\left.n=58\right)$

\begin{tabular}{lccc}
\hline GOLD Category & Number & $\mathrm{Vo}_{2}$ maxMean \pm SD & ATMean \pm SD \\
\hline Moderate & 21 & $21.28 \pm 6.17$ & $50.05 \pm 15.78$ \\
Severe & 26 & $17.37 \pm 6.32$ & $42.88 \pm 18.76$ \\
Very Severe & 11 & $13.01 \pm 4.10$ & $34.36 \pm 14.47$ \\
\hline
\end{tabular}




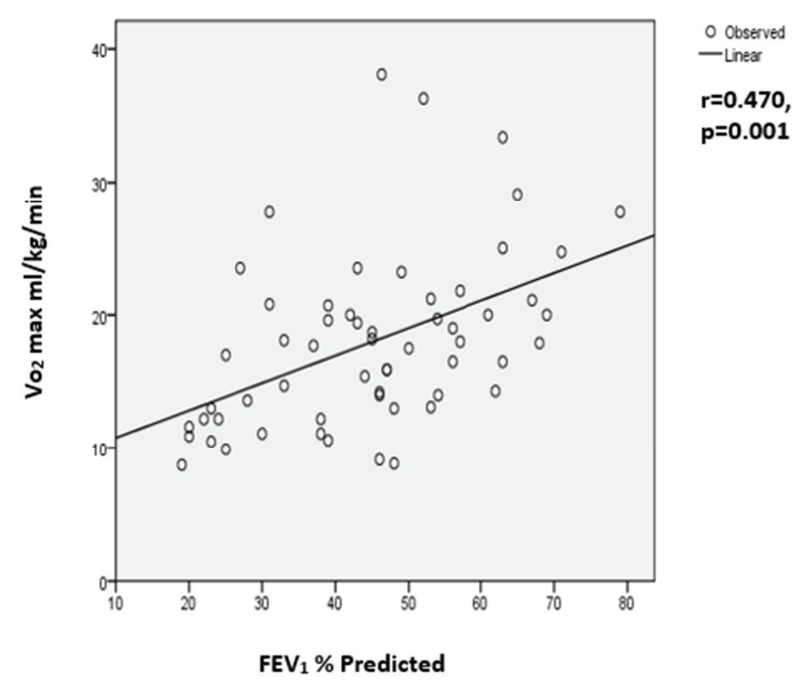

Fig.-1: Relationship between $\mathrm{Vo}_{2} \max$ and $F E V_{1}$

A significant positive correlation $(\mathrm{r}=0.47$, $\mathrm{p}=0.001$ ) between forced expiratory volume in first second ( $\mathrm{FEV}_{1}, \%$ predicted) and maximum oxygen uptake $\left(\mathrm{Vo}_{2} \max , \mathrm{ml} / \mathrm{kg} / \mathrm{min}\right)$ was observed.

Twenty-seven (46.55\%) patients with low (<40\%) AT were identified among them seven were in GOLD 2 stage, twelve were in GOLD 3 stage and eight were in GOLD 4 stage.

Majority $(34,58.62 \%)$ of patients stopped exercise due to shortness of breath; decreased oxygen saturation was the cause of stopping exercise in eight $(13.7 \%)$ patients; other causes of exercise limitation were increased heart rate, vertigo, leg fatigue, increased blood pressure, ventricular ectopic and chest tightness. Four patients were found to limit exercise due to combined shortness of breath and leg fatigue.

\section{Discussion}

Traditionally to determine the severity of disease of COPD we use $\mathrm{FEV}_{1}$. However, the present study showed that there was marked variation in functional capacity in each group of patients classified by $\mathrm{FEV}_{1}$.

We found a significant correlation between $\mathrm{FEV}_{1}$ \%predicted and $\mathrm{Vo}_{2} \max$, but there was a marked variability of $\mathrm{Vo}_{2}$ max for a given degree of airflow obstruction. That means there is a wide range of exercise performance for a given degree of airflow obstruction.
Our findings were similar to earlier observations concluding that $\mathrm{FEV}_{1}$ may not adequately characterise the degree of functional capacity impairment resulting from disease process of $\mathrm{COPD}^{10,12}$.

Ong $\mathrm{KC}$, et al.2000 in their study of clinical utility of CPET among thirty three patients found significant correlation between peak $\mathrm{Vo}_{2}$ percent predicted and $\mathrm{FEV}_{1}{ }^{12}$. In our study of fifty-eight COPD patients we also found the same type of correlation between $\mathrm{FEV}_{1} \%$ predicted and $\mathrm{Vo}_{2}$ max.

Both A. A. Ganju, et al.2010 and Ong KC, et al.2000 in their study found predominant cause for exercise limitation was dyspnoea which was consistent with our finding ${ }^{12,13}$.

In our study the second most predominant cause of exercise limitation was decrease oxygen saturation but it was leg fatigue in A. A. Ganju, et al.2010.

In evaluating AT twenty-seven (46.55\%) patients with low (<40\%) AT were identified whereas A. A. Ganju, et al.2010 found low AT in twentythree $(53.5 \%)$ COPD patients ${ }^{13}$. Among them seven were in GOLD 2 stage, twelve were in GOLD 3 stage and eight were in GOLD 4 stage. Here it shows that in every stage of disease severity classified by $\mathrm{FEV}_{1}$ contains the patients with low AT level. So, $\mathrm{FEV}_{1}$ alone cannot give the proper disease severity categorisation.

These patients with low AT during CPET have the potential to improve exercise tolerance with exercise training. This group of patients is most likely to be benefited from exercise training during pulmonary rehabilitation. Based on the exertional symptoms alone, it would not have been possible to differentiate them from rest of the COPD patients.

\section{Conclusion}

CPET is useful to measure the functional capacity in COPD patients by assessing the maximum oxygen uptake. This test is able to identify the patients who could be benefited from pulmonary rehabilitation. Moreover, it could be a good monitoring tool to see the outcome of pulmonary rehabilitation program for theseD patients. 
It can determine the cause of exercise limitation in majority of patients with COPD.

So, from our study findings we are giving emphasis to ensure more use of CPET in evaluating COPD patients. The challenges of availability and cost-effectiveness should be addressed in this regard.

\section{Limitations}

The limitation of the study was small sample size. During our study period no patients with GOLD 1 stage were identified. So, this group of patients was beyond our evaluation.

\section{References}

1. Fan VS, Ramsey SD, Make BJ, Martinez FJ. Physiologic variables and functional status independently predict COPD hospitalizations and emergency department visits in patients with severe COPD. COPD. 2007; 4(1) : 29-39

2. Pitta F, Troosters T, Probst VS, Spruit MA, Decramer M, Gosselink R. Physical activity and hospitalization for exacerbation of COPD. Chest. $2006 ; 129(3)$ : $536-44$

3. Garcia-Aymerich J, Lange P, Benet M, Schohr P, Anto JM. Regular physical activity reduces hospital admission and mortality in chronic obstructive pulmonary disease: a population based cohort study. Thorax . 2006; 61(9):772-8

4. American Thoracic Society. Pulmonary rehabilitation: American Thoracic Society. Am J Respir Crit Care Med. 1999;159:1666-1682.

5. AmericanAssociation of Cardiovascular and PulmonaryRehabilitation. Pulmonary rehabilitation: joint ACCP/AACVPR evidence-based guidelines. ACCP/AACVPR Pulmonary Rehabilitation Guidelines Panel. Chest. 1997;112:1363-1396.

6. Palange $\mathrm{P}$, Ward SA, Carlse K-H, Casaburi R, Gallagher CG, Gosselinke R, O'Donnell DE,PuenteMaestu L, Schols AM, Sing S, Whipp BJ. Recommendations on the use of exercise testing in clinical practice. EurRespir J. 2007;29(1):185-209

7. ATS statement: Guide line for the six-minute walk test. Am J Respir Crit Care Med 2002;166(1):111-17

8. Ozalevlis S, Ozden A, Hill O, Akkoclu A. Comparison of the Sit to Stand Test with 6 min walk test in patients with chronic obstructive pulmonary disease. Respir Med. 2007;101(2):286-93

9. Brunelli A, AI Refai M, Monteverde M, Borri A, Salati M, Fianchini A. Stair climbing test predicts cardiopulmonary complications after lung resection. Chest. 2002;121(4):1106-10

10. Ortega F, Montemayor T, Sánchez A, Cabello F, Castollo J. A Role of cardiopulmonary exercise testing and the criteria used to determine disability in patients with severe chronic obstructive pulmonary disease. Am J Respir Crit Care Med. 1994;150:74751 .

11. Miller MR, Hankinson J, Brusasco V, Burgos F, et al. ATS/ERS Task Force. Standardisation of Spirometry. EurRespir J. 2005 ;26:319-38

12. Ong KC, Ong YY. Cardiopulmonary exercise testing in patients with chronic obstructive pulmonary disease. Ann Acad Med Singapore. 2000;29:648-52.

13. Ganju A. A., Fuladi A. B., Tayade B. O., Ganju N. A. Cardiopulmonary Exercise Testing in Evaluation of Patients of Chronic Obstructive Pulmonary Disease. Indian J Chest Dis Allied Sci. 2011;53:87-91 